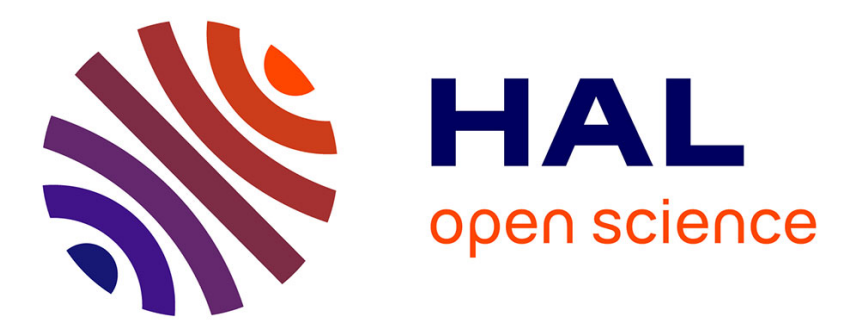

\title{
A comprehensive study of the use of temporal moments in time-resolved diffuse optical tomography: part II. Three-dimensional reconstructions
}

Nicolas Ducros, Anabela da Silva, Lionel Hervé, Jean-Marc Dinten, Françoise Peyrin

\section{To cite this version:}

Nicolas Ducros, Anabela da Silva, Lionel Hervé, Jean-Marc Dinten, Françoise Peyrin. A comprehensive study of the use of temporal moments in time-resolved diffuse optical tomography: part II. Three-dimensional reconstructions. Physics in Medicine and Biology, 2009, 54 (23), pp.7107-7119. 10.1088/0031-9155/54/23/005. hal-00872899

\section{HAL Id: hal-00872899 \\ https://hal.science/hal-00872899}

Submitted on 18 Oct 2013

HAL is a multi-disciplinary open access archive for the deposit and dissemination of scientific research documents, whether they are published or not. The documents may come from teaching and research institutions in France or abroad, or from public or private research centers.
L'archive ouverte pluridisciplinaire HAL, est destinée au dépôt et à la diffusion de documents scientifiques de niveau recherche, publiés ou non, émanant des établissements d'enseignement et de recherche français ou étrangers, des laboratoires publics ou privés. 


\title{
A comprehensive study of the use of temporal moments in Time-Resolved Diffuse Optical Tomography: Part II. Three dimensional reconstructions
}

\author{
Nicolas Ducros ${ }^{1,2}$, Anabela Da Silva ${ }^{3}$, Lionel Hervé ${ }^{1}$, \\ Jean-Marc Dinten ${ }^{1}$, and Françoise Peyrin ${ }^{2}$ \\ ${ }^{1}$ CEA, LETI, MINATEC, 17 rue des Martyrs, F-38054 Grenoble, France. \\ 2 CREATIS-LRMN, INSERM U 630; CNRS UMR 5220; Université de Lyon; \\ INSA de Lyon, bât. Blaise Pascal, F-69621 Villeurbanne Cedex, France. \\ ${ }^{3}$ Institut Fresnel, Université Aix-Marseille; CNRS UMR 6133; École Centrale \\ Marseille, Campus universitaire de Saint-Jérôme, F-13013 Marseille. \\ E-mail: nicolas.ducros@cea.fr
}

\begin{abstract}
The present paper addresses the inverse problem of time-resolved (fluorescence) diffuse optical tomography from temporal moments of the measurements. A methodology that enables to provide fairly comparable reconstructions is presented. The proposed reconstruction methodology is applied to infinite medium synthetic phantoms in the transmission geometry. Reconstructions are performed for moment orders increasing from 0 to 3 . The reconstruction quality is shown to be increasing when higher moment orders are added. However, the value of the highest useful moments order strongly depends on the number of photons that can be acquired. In particular, it can be considered that the benefit of using higher order moments vanishes when fewer than $10^{8}$ photons are detected. The evolution of the reconstruction quality with respect to the optical properties of the medium and fluorescence lifetime is also shown.
\end{abstract}




\section{Introduction}

Diffuse Optical Tomography (DOT) and Fluorescence DOT (FDOT) are two techniques aiming at exploring the inner structure of biological tissues with light in the near infra-red range. DOT deals with the determination of the optical properties of the medium while FDOT deals with the detection of fluorescent markers. The principle of these methods is to process the light exiting the medium at different detector positions for a set of source positions and to solve an inverse problem to reconstruct 3-dimensional (3-D) maps of the parameters of interest.

When available, the time-domain (TD) light measurements are commonly reduced to their moments up to a given order. In a companion paper, the theoretical aspects of calculating the moments the forward problem of FDOT have been investigated. Analytical expressions of the moments of the forward model have been derived for any order, as well as a moment-based noise model. In the present paper, the inverse problem is addressed. Our purpose is to accurately quantify the benefit of using higher order moments and determine, in terms of reconstruction quality, the situations for which the moment method is the more profitable. Toward this end, an infinite and homogeneous medium injected with a distribution of local fluorescent markers is considered. Synthetic TD measurements are simulated in the transmission geometry, i.e. light sources and detectors are placed on either side of the object to be imaged. Then, reconstructions of the markers concentration are performed for moment orders ranging from 0 to 3 .

In FDOT, the markers concentration within the medium can be assumed to be linearly related with the measurements. This hypothesis holds provided that the absorption of the medium is larger than the absorption of the markers, which is generally the case in practical scenarios. From physical considerations, it is possible to derive the so-called forward model that maps the measurement onto the marker concentration. The building of the forward model, when moments of the TD measurements are considered, has been described in the first part of this work (Ducros et al., 2009).

In section 2, we briefly present our formalism. Reconstructing the markers concentration from the measurements is addressed in the resolution of the so-called inverse problem. Since the inverse problem in FDOT is ill-posed, meaning that the reconstruction is highly sensitive to some perturbations in the measurements, the reconstruction has to be performed within the framework of regularization. An extensive literature can be found on the subject and several families of algorithms are available (Bertero et al., 1988). A brief review of the regularization strategies completes the section 2 .

In section 3, we describe the synthetic phantoms under study as well as the Poisson-based noise model that has been used.

It should be emphasized that in the context of this study, dealing with illposedness is a necessary condition but not a sufficient one. Indeed, to provide fairly comparable reconstructions, the reconstruction procedure must ensure that the regularization is strictly equivalent from reconstruction to reconstruction. A particular care must be taken to fulfil this condition since the measurements are of different nature (different order moments have different dimensions) and sensitivity to noise (higher order moments are more sensitive to noise). A number of general methods, among them the discrepancy principle (Galatsanos and Katsaggelos, 1992), the L-curve (Hansen, 1992), the generalized cross validation (Golub et al., 1979), are 
available to cope with the problem of regularization control (see Kilmer and O'Leary. (2001) for an overview of the different approaches). Unfortunately, it has been noticed that these general methods can lead to over-regularization in the context of DOT (Culver et al., 2003). Hence, empirical methods based on object contrast (Culver et al., 2003) or visual assessment (Graves et al., 2004; Selb et al., 2007; Kumar et al., 2008) are commonly employed and show good practical results. However, the use of empirical methods prevents from ensuring the comparability between reconstructions.

In section 4 we present a original methodology that allows for controlling thoroughly the regularization while avoiding the undesirable over-regularization. It is thought that the proposed reconstruction methodology could provide a reconstruction framework well adapted to comparative studies.

The section 5 provides the reconstructed concentrations from noisy synthetic moments, increasing the considered orders from 0 to 3 , for several configurations. The influence of three kinds of parameters is investigated. The more critical parameter is found to be the number of detected photons that sets the signal-to-noise ratio (SNR). The influence of the optical properties of the medium as well as of the lifetime of the fluorescent marker is also shown.

\section{Theoretical background}

\subsection{Notations}

Matrices are marked with bold capitals while vectors are marked with bold minuscules. Vectors are seen as column-matrices: $\mathbf{x}=\left(x_{1}, \ldots, x_{n}\right)^{T}$. The notation $\|\cdot\|$ denotes the discrete $l_{2}$ norm defined by $\|\mathbf{x}\|=\left(\mathbf{x}^{T} \mathbf{x}\right)^{1 / 2}$. We note $\|\cdot\|_{\mathbf{L}}$, the weighted $\mathrm{l}_{2}$ norm induced by the positive-definite matrix $\mathbf{L}$ and defined by: $\|\mathbf{x}\|_{\mathbf{L}}=\left(\mathbf{x}^{T} \mathbf{L}^{T} \mathbf{L x}\right)^{1 / 2}$.

\subsection{Forward problem: moment-based formalism}

Let us consider a fluorescence marker distribution $\mathbf{c}$ within the medium. The vector $\mathbf{c}=\left(c_{1}, \ldots, c_{N}\right)^{T}$ is composed of the local concentration $c_{n}$ at positions $\left\{\mathbf{r}_{\mathbf{n}}\right\}_{n=1 \ldots N}$. The medium is probed by a set of light sources at positions $\{\mathbf{s}\}$ and a set of detectors at positions $\{\mathbf{d}\}$. In the following, I source-detector pairs $\left\{\left(\mathbf{s}_{i}, \mathbf{d}_{i}\right)\right\}$ are considered. The light detected at position $\mathbf{d}_{i}$ due to excitation at position $\mathbf{s}_{i}$ is noted $u_{i}(t)=u\left(\mathbf{s}_{i}, \mathbf{d}_{i}, t\right)$. It originates from the superposition of all the local fluorescence light contributions. Mathematically we have the following weighted sum: $u_{i}(t)=\sum_{n=1}^{N} w_{n}^{i}(t) c_{n}$. Regarding the whole set of light measurements $\mathbf{u}(t)=\left(u_{1}(t), \ldots, u_{I}(t)\right)^{T}$ a matrix formalism can be adopted. It leads to

$$
\mathbf{u}(t)=\mathbf{W}(t) \mathbf{c},
$$

where $\mathbf{W}(t)=\left(w_{n}^{i}(t)\right)_{i, n}$ is the TD forward model operator that maps the local concentrations onto the light measurements. The building of the matrix $\mathbf{W}(t)$, usually referred to as the TD weight matrix, has been reported in a companion paper (Ducros et al., 2009). Now, we consider the temporal moments of the TD forward model given in (1). The $k^{\text {th }}$ order moments of a signal $f$ is defined consistently with Ducros et al. (2009) by: $m_{k}\{f(t)\}=\int_{0}^{\infty} f(t) t^{k} \mathrm{~d} t$. Then, the moment transformation can be applied component-wise on the linear system (1), which leads to

$$
\mathbf{u}[k]=\mathbf{W}[k] \mathbf{c},
$$


where $\mathbf{u}[k]=m_{k}\{\mathbf{u}(t)\}$ is the $k^{\text {th }}$ order moments of the measurements and $\mathbf{W}[k]=$ $m_{k}\{\mathbf{W}(t)\}$ is the $k^{\text {th }}$ order moments of the TD weight matrix. In the first paper of this serie, the analytical expression for the entries of $\mathbf{W}[k]$ is given for an infinite homogeneous medium (refer to equation 14 and 17 of Ducros et al. (2009)).

\subsection{Inverse problem: regularization strategies}

The inverse problem in FDOT consists in determining the concentration vector $\mathbf{c}$ from the measurement vector $\mathbf{u}$ and the knowledge of the weight matrix $\mathbf{W}$. Unfortunately, the inverse problem in FDOT is well-known to be ill-posed, meaning that a small deviation $\delta \mathbf{u}$ in the measurement $\mathbf{u}$ - practically speaking the presence of noise leads to a large deviation $\delta \mathbf{c}$ in the reconstructed concentration c. Formally, the amplification of the measurement deviation is given by (Regińska, 2004)

$$
\frac{\|\delta \mathbf{c}\|}{\|\mathbf{c}\|} \leq \kappa(\mathbf{W}) \frac{\|\delta \mathbf{u}\|}{\|\mathbf{u}\|}
$$

where $\kappa(\mathbf{W})$ is the condition number of the matrix of the forward model. In this study, for instance, $\kappa(\mathbf{W})$ is always larger than $10^{8}$ explaining that even a small measurement deviation can lead to a large reconstruction deviation. To reduce the influence of noise and stabilize the inversion, regularization schemes must be introduced. We categorized them into two groups: the implicit regularization schemes and the explicit regularization schemes.

The implicit regularization scheme involves minimizing the classical least square cost function

$$
\Phi(\mathbf{c})=\|\mathbf{u}-\mathbf{W} \mathbf{c}\|^{2} .
$$

The regularization is performed by minimizing (4) with some iterative algorithms. The algebraic reconstruction technique has been used in a large number of instances (Gaudette et al., 2000; Lam et al., 2005; Gao et al., 2002, 2006; Nielsen et al., 2009) as well as other classical optimization approaches as Landweber algorithm, steepest descent or conjugate gradient descent (Gaudette et al., 2000). Then, the regularization strategy consists in stopping the retained algorithm before convergence to the minimum of (4).

In the explicit regularization schemes, the cost function (4) is modified by adding a regularization term, weighted by the so-called regularization parameter. In the classical Tikhonov approach the regularization term is chosen to be the square of the norm of the concentration. Mathematically, the Tikhonov cost function is (Tikhonov and Arsenin, 1977)

$$
\Phi^{\alpha}(\mathbf{c})=\|\mathbf{u}-\mathbf{W} \mathbf{c}\|^{2}+\alpha\|\mathbf{c}\|^{2},
$$

$\alpha$ being the regularization parameter. The solution $\mathbf{c}=\operatorname{argmin} \Phi^{\alpha}(\mathbf{c})$ strongly depends on $\alpha$. Indeed, let us consider the two extreme scenarios: $\alpha=0$ and $\alpha=\infty$. If $\alpha=0$, then no regularization is performed and the resulting solution $\mathbf{c}(\alpha=0)$ is dominated by noise and thus highly unstable. If $\alpha=\infty$, the inverse problem is reduced to minimizing the energy of the concentration $\|\mathbf{c}\|^{2}$. This leads to the highly stable - or noise-free - solution $\mathbf{c}(\alpha=\infty)=\mathbf{0}$. This meaningless solution is said to be over-smoothed since its energy is zero. In section 4 , the key choice of $\alpha$ as a compromise between instability and over-smoothing is further discussed. 


\section{Numerical Experiments}

\subsection{Description of the numerical phantoms}

We consider the homogeneous infinite medium phantom represented on figure 1 . The refractive index $n$ of the medium is set to 1.4. Different absorption coefficients $\mu_{a}$ and the reduced scattering coefficients $\mu_{s}^{\prime}$ are investigated. The study ranges $\mu_{a} \in[0.01,0.5] \mathrm{cm}^{-1}$ and $\mu_{s}^{\prime} \in[1,20] \mathrm{cm}^{-1}$ - should cover most of the practical situations. For the reconstruction purpose, the medium is evenly discretized. The resulting 810 cubic voxels of size $(0.5 \mathrm{~cm})^{3}$ span a total volume of $4.5 \times 4.5 \times 5 \mathrm{~cm}^{3}$. The medium is probed by 12 light sources and 12 light detectors, resulting in a set of 144 source-detector pairs. Sources and detectors are both evenly arranged within two circular disks of radius $1.5 \mathrm{~cm}$. The two disks are located $5 \mathrm{~cm}$ apart, surrounding the medium in the so-called transmission geometry.

We regard fluorescent makers with lifetime $\tau$ in the range $\tau \in[0,5]$ ns. The lower bound $\tau=0$ ns can stand for the problem of absorption reconstruction in DOT, while the upper bound $\tau=5$ ns can describe the use of long lifetime markers such as quantum dots (Medintz et al., 2005). The phantom under study contains three tumoral zones where the fluorescent markers concentrate (see figure 1 for illustration). The three tumours are centred at the positions $\mathbf{r}_{1}=[-1.5,1,2.25] \mathrm{cm}$ (tumor $\# 1$ ), $\mathbf{r}_{2}=[1,1,3.75] \mathrm{cm}$ (tumor \#2) and $\mathbf{r}_{3}=[1,1,1.25] \mathrm{cm}$ (tumor \#3). This configuration is not easy to deal with for two reasons. First, the tumour \#1 is located far away from both source and detector plans, within a zone of poor sensitivity (Kepshire et al., 2007). Second, the tumours \#2 and \#3 are aligned along the $\mathrm{z}$-axis for which FDOT exhibits low ability to separating two adjacent inclusions (Gao et al., 2002). To simulate the non-specificity of the makers, a homogeneous fluorescent background is also considered. While targeted markers typically offer a tumour-to-background ratio of 5:1, recent works have shown that the tumour-to-background ratio of activable markers can reach 200:1 (Urano et al., 2009). In this study, the tumour-to-background ratio is set to $40: 1$.

\subsection{Noise model}

Classically, the noise in optical measurements is assumed to follow a Poisson distribution. Recently, this model has been complexified to take into considerations the specificities of FDOT measurements (Hyde et al., 2007). In this study the rate of detected photons is hypothesized to be large enough to neglect any other sources of noise and notably the electronic (thermal) noise. Furthermore, under the Poisson distribution can be modelled to good approximation by a Gaussian distribution. Within this framework, any noisy measurement $\tilde{u}(t)$ is given by

$$
\tilde{u}(t)=u(t)+\mathcal{N}\left\{\mu=0, \sigma^{2}=u(t)\right\},
$$

where $\mathcal{N}\left(\mu, \sigma^{2}\right)$ denotes for a realization of a Gaussian random variable of mean $\mu$ and variance $\sigma^{2}$. In a companion paper we have shown how noise on a TD signal corrupts its moments (see equation 20 of Ducros et al. (2009)). Using this result we have:

$$
m_{k}[\tilde{u}]=m_{k}[u]+\mathcal{N}\left\{\mu=0, \sigma^{2}=m_{2 k}[u]\right\} .
$$

Consistently with the notations developed in the section 2 , the vector $\tilde{\mathbf{u}}[k]=$ $\left(m_{k}\left\{\tilde{u}_{1}(t)\right\}, \ldots, m_{k}\left\{\tilde{u}_{I}(t)\right\}\right)^{T}$ denotes $k^{\text {th }}$ order moments of the noisy measurements. 


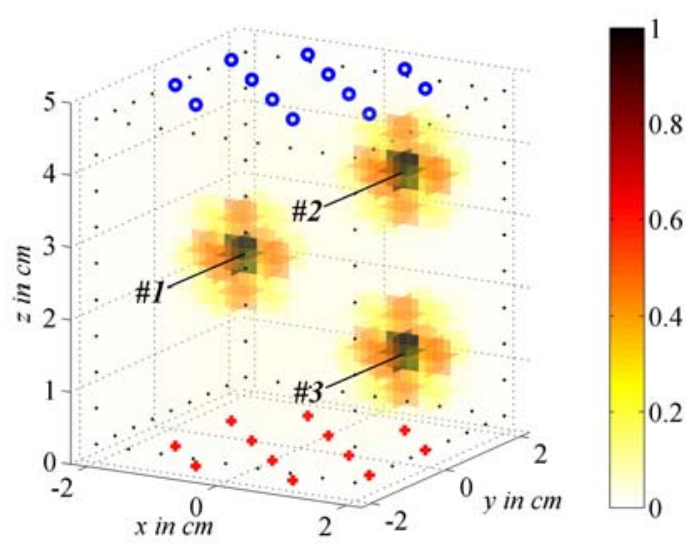

Figure 1. Numerical phantom used for the reconstructions. The blue circles indicate the detectors, the red crosses indicate the sources and the black dots indicate the contour of the mesh. The marker concentration within the medium is plotted with the hot colour scale

For a measurement $u(t)$ corrupted by a Poisson noise, the standard deviation of the noisy measurement is $\sqrt{u(t)}$. The SNR, expressed in $\mathrm{dB}$, is then $20 \log (u)$, meaning that the value of $u$ fixes the SNR. The larger the measurement $u$ is, the better the SNR. In the following, the SNR is tuned by rescaling the moments with an appropriate constant. The constant is chosen so as to fix the largest moment of order 0 to a desired maximal count of detected photons $C_{\max }$. This mathematical operation is experimentally equivalent to increasing the time of integration until $C_{\max }$ photons are collected on one of the detectors. In the present study, $C_{\max }$ covers the range $\left[10^{6}, 10^{14}\right]$ photons.

\section{Reconstruction procedure}

In this part, we consider the reconstruction of the concentration vector from the moments up to a given order. Let $K$ be highest selected moments order. Let $\tilde{\mathbf{u}}_{K}=(\tilde{\mathbf{u}}[0], \ldots, \tilde{\mathbf{u}}[K])^{T}$ be the stack of the moments of the measurements up to order $K$ and $\mathbf{W}_{K}=(\mathbf{W}[0], \ldots, \mathbf{W}[K])^{T}$ be the stack of the moments of the TD weight matrix up to order $K$. The reconstructed concentration sought from the moments up to order $K$ is noted $\mathbf{c}_{K}$.

\subsection{Choice of the algorithm}

To benefit from thorough control of the regularization, the choice is made to consider an explicit regularization scheme and thus to minimize the following cost function:

$$
\Phi^{\alpha}(\mathbf{c})=\left\|\tilde{\mathbf{u}}_{K}-\mathbf{W}_{K} \mathbf{c}\right\|_{\mathbf{L}}^{2}+\alpha\|\mathbf{c}\|^{2} .
$$

Here $\mathbf{L}$ is a diagonal matrix chosen to penalize the noisier measurements. Explicitly, the $j^{\text {th }}$ diagonal entry of $\mathbf{L}$ is the standard deviation of the $j^{\text {th }}$ component of $\tilde{\mathbf{u}}_{K}$. Advantageously, the choice of this weighted norm results in considering dimensionless moments and resolves the problem of dealing with measurements of different nature. 
Let $\mathbf{c}_{K}(\alpha)$ be the reconstructed concentration using moments up to order $K$. $\mathbf{c}_{K}(\alpha)$ is the minimiser of the Tikhonov cost function given by (8). It can be obtained either analytically or by iterative algorithms. Here, the analytical method is preferred since it avoids the problems of convergence and the determination of the stopping criterion. Explicitly, $\mathbf{c}_{K}(\alpha)$ is given by

$$
\mathbf{c}_{K}(\alpha)=\left(\mathbf{W}_{K}^{T} \mathbf{L}^{T} \mathbf{L} \mathbf{W}_{K}+\alpha \mathbf{I}_{N}\right)^{-1} \mathbf{W}_{K}^{T} \mathbf{L}^{T} \tilde{\mathbf{u}}_{K}
$$

For conciseness, we note: $\mathbf{c}_{K}(\alpha)=\mathbf{W}_{K}^{\alpha} \tilde{\mathbf{u}}_{K}$ where the Moore-Penrose pseudo inverse $\mathbf{W}_{K}^{\alpha}$ is defined accordingly to (9). Interestingly, $\mathbf{W}_{K}^{\alpha}$ can be computed from the singular value decomposition (SVD) of $\mathbf{L W}_{K}$. The SVD consists of writing $\mathbf{L W}_{K}$ as $\mathbf{L} \mathbf{W}_{K}=\mathbf{U} \boldsymbol{\Sigma} \mathbf{V}^{T}$, where $\mathbf{U}$ and $\mathbf{V}$ are two orthonormal matrices and $\boldsymbol{\Sigma}$ is a diagonal matrix. Substituting this decomposition in (9), it can be seen that

$$
\mathbf{c}_{K}(\alpha)=\mathbf{V} \boldsymbol{\Sigma}\left(\boldsymbol{\Sigma}^{2}+\alpha \mathbf{I}\right)^{-1} \mathbf{U}^{T} \tilde{\mathbf{u}}_{K}
$$

where I stands for the identity matrix (see section 2 of Hansen (1987) for details). Practically, $\mathbf{c}_{K}(\alpha)$ is obtained from the following steps: 1) SVD of $\mathbf{L W}_{K}$ including the storage of $\mathbf{U}, \boldsymbol{\Sigma}$, and $\mathbf{V}, 2)$ inversion of the diagonal matrix $\boldsymbol{\Sigma}^{2}+\alpha \mathbf{I}$, and 3) left multiplication of the result by $\mathbf{V} \boldsymbol{\Sigma}$ and right multiplication by $\mathbf{U}^{T} \tilde{\mathbf{u}}_{K}$. In this process, the SVD is the time-consuming step. However, once the SVD has been performed, the computation of (9) for multiple regularization parameters $\alpha$ and measurements vector $\tilde{\mathbf{u}}_{K}$ is almost done on the fly. Indeed, $\alpha$ and $\tilde{\mathbf{u}}_{K}$ are only involved in steps 2 and 3 , whose computation is very fast.

\subsection{Determination of the regularization parameter}

The approach detailed below fixes the regularization parameter from the definition of some acceptable reconstruction variability. To illustrate the philosophy of the proposition, let us consider a very simple example. Let $\tilde{\mathbf{u}}_{K}^{(1)}$ be a given noisy measurement vector. The concentration vector reconstructed from this noisy measurement is $\tilde{\mathbf{c}}_{K}^{(1)}(\alpha)=\mathbf{W}_{K}^{\alpha} \tilde{\mathbf{u}}_{K}^{(1)}$. If the experiment is reproduced in the same conditions, then a new noisy measurement vector $\tilde{\mathbf{u}}_{K}^{(2)}$ is acquired. The resulting reconstructed concentration is now $\tilde{\mathbf{c}}_{K}^{(2)}(\alpha)=\mathbf{W}^{\alpha} \tilde{\mathbf{u}}^{(2)}$. It is reasonable to expect that an appropriate $\alpha=\alpha^{*}$ can ensure $\tilde{\mathbf{c}}_{K}^{(1)}\left(\alpha^{*}\right)$ to be close to $\tilde{\mathbf{c}}_{K}^{(2)}\left(\alpha^{*}\right)$.

To estimate the variability of the reconstructions, we first define the mean reconstruction vector $\mathrm{E}\left[\tilde{\mathbf{c}}_{K}(\alpha)\right]$ and the standard deviation reconstruction vector $\delta\left[\tilde{\mathbf{c}}_{K}(\alpha)\right]$. Explicitly, from a set of $\Omega$ reconstructed concentration vectors, the two quantities are computed according to the two classical non-biased estimators:

$$
\mathrm{E}\left[\tilde{\mathbf{c}}_{K}(\alpha)\right]=\frac{1}{\Omega} \sum_{\omega=1}^{\Omega} \mathbf{c}_{K}^{(\omega)}, \quad \text { and } \quad \delta\left[\tilde{\mathbf{c}}_{K}(\alpha)\right]=\left(\frac{1}{\Omega-1} \sum_{\omega=1}^{\Omega}\left\{\tilde{\mathbf{c}}_{K}^{(\omega)}-\mathrm{E}\left[\tilde{\mathbf{c}}_{K}(\alpha)\right]\right\}^{2}\right)^{1 / 2} .
$$

With synthetic experiments, for which the noiseless measurements are available, only the standard deviation has to be calculated. Indeed, the mean reconstruction vector equals the noiseless reconstruction vector $\mathbf{c}_{K}(\alpha)=\mathbf{W}_{K}^{\alpha} \mathbf{u}_{K}$.

Then, the reconstruction variability $\mathcal{V}$ is defined on the model of (3):

$$
\mathcal{V}(\alpha)=\frac{\left\|\delta\left[\tilde{\mathbf{c}}_{K}(\alpha)\right]\right\|}{\left\|\mathbf{c}_{K}(\alpha)\right\|}=\frac{\left\|\delta\left[\tilde{\mathbf{c}}_{K}(\alpha)\right]\right\|}{\left\|\mathrm{E}\left[\tilde{\mathbf{c}}_{K}(\alpha)\right]\right\|} .
$$



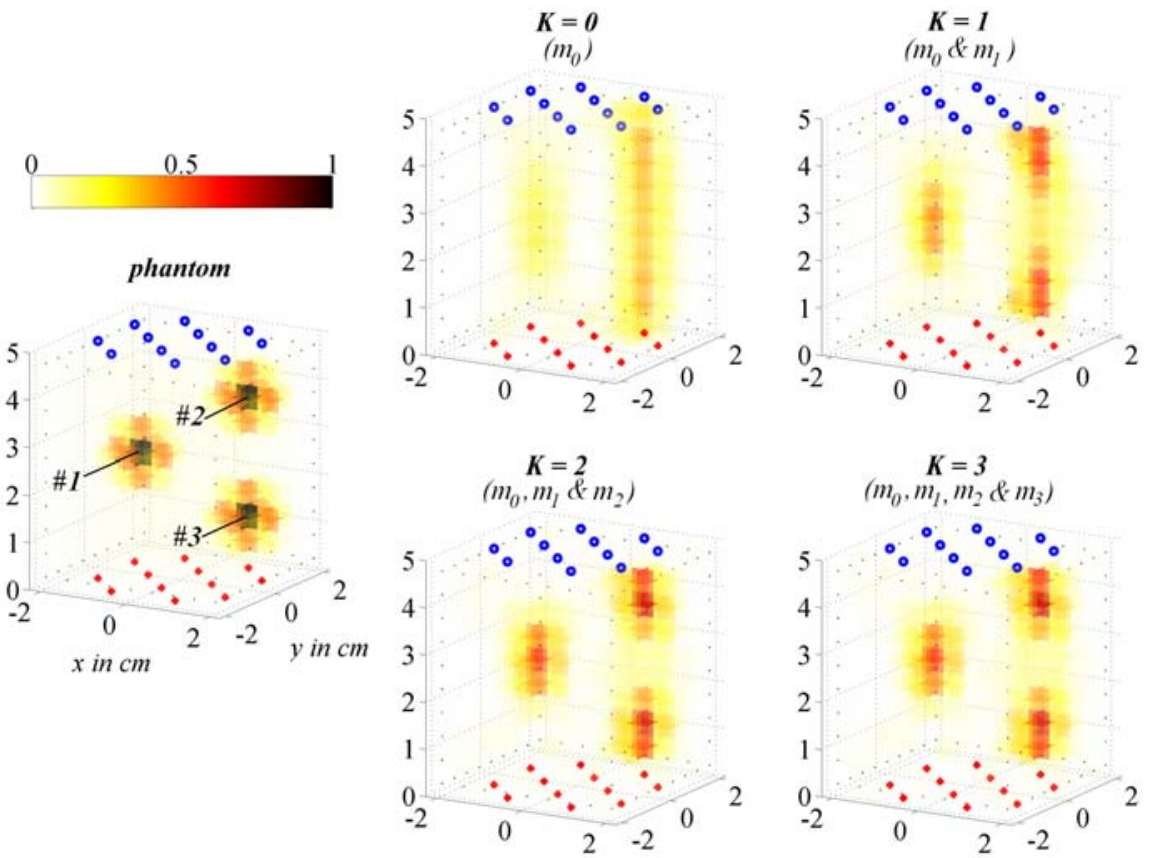

Figure 2. 3-D reconstructions from moments up to order $K$ of $0,1,2$ and 3 (right) to be compared to the phantom (left). Here, $\mu_{a}=0.1 \mathrm{~cm}^{-1}, \mu_{s}^{\prime}=$ $10 \mathrm{~cm}^{-1}$ and $C_{\max }=10^{14}$ photons.

Setting $\Omega$ to $50, \mathcal{V}(\alpha)$ was computed for multiple $\alpha$ 's and it was observed that $\mathcal{V}(\alpha)$ decreases monotonically. For $\alpha^{*}$ such that $\mathcal{V}\left(\alpha^{*}\right)=0.1$, the reconstructions exhibit both limited instability and over-smoothing. Therefore, the retained $\alpha^{*}$ 's are chosen by dichotomy to verify $\mathcal{V}\left(\alpha^{*}\right)=0.1$.

It can be noted that the proposed procedure for choosing $\alpha$ differs from the so-called Miller criterion (Miller, 1970). The Miller criterion sets $\alpha$ as $\alpha^{*}=\epsilon^{2} / E^{2}$, where $\epsilon$ stands for the upper bounds of $\|\mathbf{u}-\mathbf{W} \mathbf{c}\|^{2}$ and $E$ for the upper bound of $\|\mathbf{c}\|^{2}$. Here the constraint is put on the variability of the reconstruction and not on the smoothness of the reconstruction that is a priori unknown in experimental scenarios.

\section{Results and discussion}

An example of the 3-D concentration reconstructions performed from moments up to different orders (for different $K$ ) is presented on figure 2. In this illustration, $\mu_{a}$ is set to $0.1 \mathrm{~cm}^{-1}, \mu_{s}^{\prime}$ to $10 \mathrm{~cm}^{-1}, \tau$ to $0 \mathrm{~ns}$ and $C_{\max }$ to $10^{14}$ photons. The aforementioned optical properties are commonly encountered in FDOT problems; they are notably in agreement with in vivo values of breast tissue (Cheong et al., 1990).

From this first reconstruction example, it can be seen how the use of higher order moments improve the reconstruction quality. Using only the $0^{\text {th }}$ order moments - the $m_{0}$ 's in short - results in a poor separability of tumour \#2 and \#3. Now let us compare the cases $K=0$ and $K=1$. When the $m_{1}$ 's are added to the $m_{0}$ 's, the presence of tumour \#1 is exhibited. In parallel, a better separation of the tumours \#2 and \#3 is 

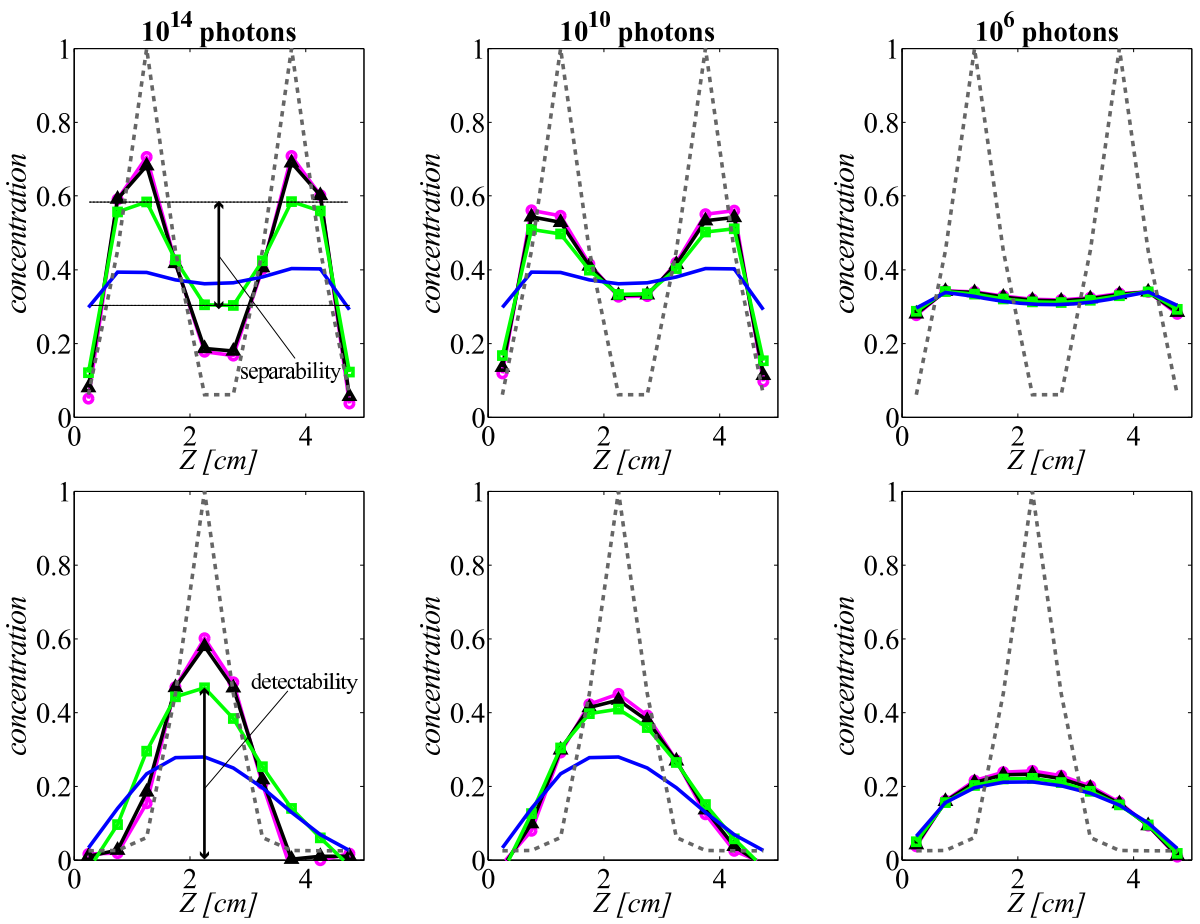

Figure 3. Reconstruction profiles along the z-axis, through tumor \#2 and \#3 (first row) and through tumor \#3 (second row). The dashed lines indicate the ground truth concentration profiles. In the first column $C_{\max }$ is set to $10^{14}$, in the second to $10^{10}$ and in the third to $10^{6}$. Moments are used up to order 0 (blue solid line), 1 (green marked with $\square$ ), 2 (black marked with $\Delta$ ) and 3 (magenta marked with $\bigcirc$ ). Here, $\mu_{a}=0.1 \mathrm{~cm}^{-1}, \mu_{s}^{\prime}=10 \mathrm{~cm}^{-1}$.

observed. The detectability of tumour \#1 as well as the separability between tumour \#2 and \#3 are further enhanced when the $m_{2}$ 's are adjoined to the $m_{0}$ 's and the $m_{1}$ 's (consider the case $K=2$ ). However, the difference between the reconstruction for $K=2$ and $K=3$ can hardly be told. In this example, it can be concluded that higher order moments provide extra information in terms of reconstruction quality up to order 2 .

\subsection{Influence of the signal-to-noise ratio}

On figure 3, reconstructed concentration profiles along the $z$-axis are depicted for 3 maximum numbers of detected photons. The profiles through tumour \#1 and through tumour $\# 2$ and $\# 3$ are represented for $C_{\max }=10^{14}$ photons, $C_{\max }=10^{10}$ photons, and $C_{\max }=10^{6}$ photons.

We have identified the number of detected photons - equivalently the SNR - to be the most critical parameter in terms of reconstruction quality. As shown on the reconstruction profiles of figure 3 , the benefit of using higher order can vary from very significant to null depending on the number of photons that can be detected. When $C_{\max }=10^{6}$ photons, no benefit in using higher order moments was observed whatever the optical properties of the medium and the fluorescence lifetime. Benefits of using higher order only appears for $C_{\max }$ larger $10^{8}$ detected photons. Experimentally 

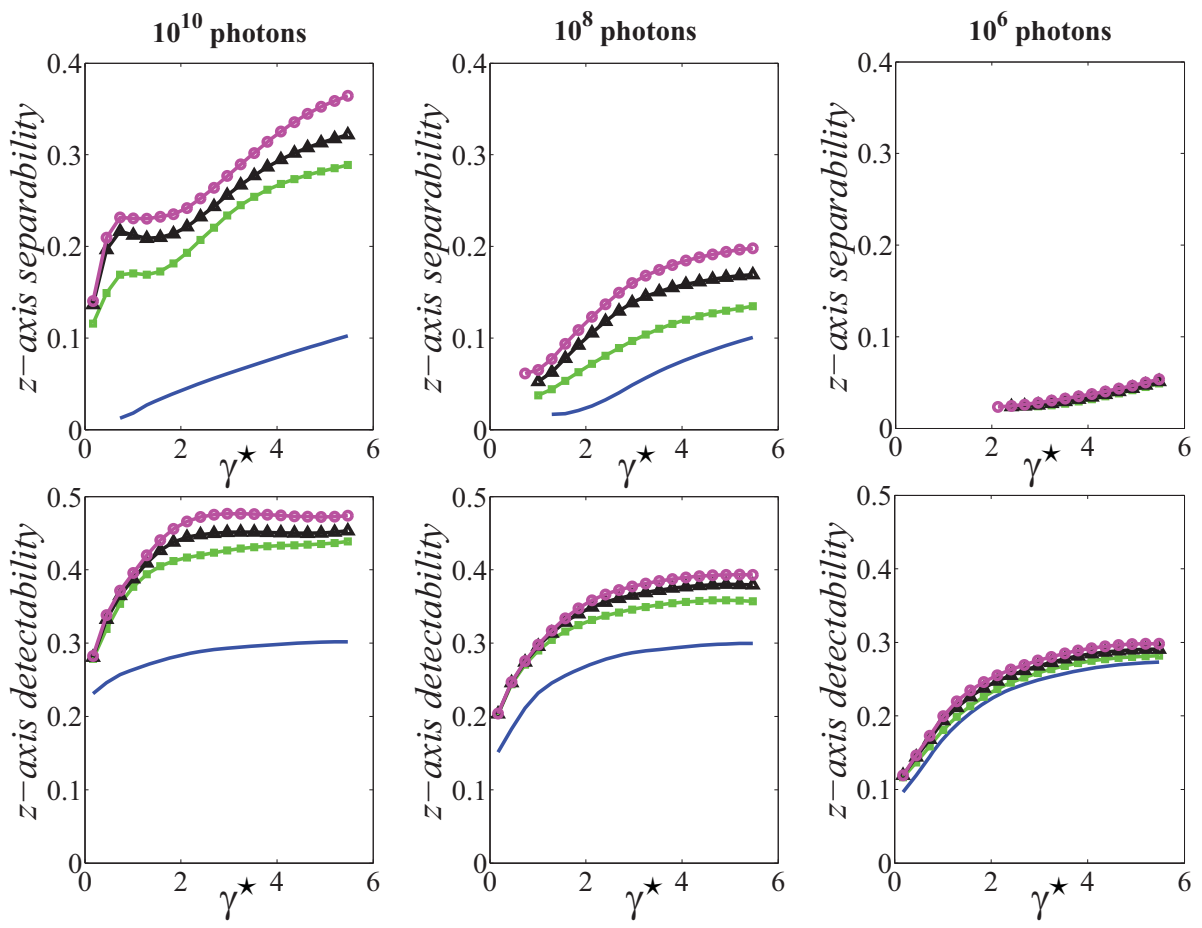

Figure 4. Sensitivity and separability along the z-axis for varying optical properties. In the first column $C_{\max }$ is set to $10^{10}$, in the second to $10^{8}$ and in the third to $10^{6}$. Moments are used up to order 0 (blue solid line), 1 (green marked with $\square$ ), 2 (black marked with $\triangle$ ) and 3 (magenta marked with $\bigcirc$ ).

speaking this conclusion is of the highest importance since such level of detection may hardly be reached.

\subsection{Influence of the optical properties of the medium}

In this part we assess the influence of the optical properties on the reconstruction quality. In terms of optical properties, we have previously identified the wave number $\gamma^{\star}=\left(3 \mu_{a} \mu_{s}^{\prime}\right)^{1 / 2}$ to be the driving parameter when the lifetime is zero (refer to Part I). Hence, the reconstruction quality for a varying $\gamma^{\star}$ is evaluated. Small $\gamma^{\star}$ represent low absorbing and diffusing media while large $\gamma^{\star}$ represent highly absorbing and diffusing media. The smallest $\gamma^{\star}$ is obtained with $\mu_{a}=0.01 \mathrm{~cm}^{-1}$ and $\mu_{s}^{\prime}=1 \mathrm{~cm}^{-1}$. The largest $\gamma^{\star}$ is obtained with $\mu_{a}=0.5 \mathrm{~cm}^{-1}$ and $\mu_{s}^{\prime}=20 \mathrm{~cm}^{-1}$.

To summarize the reconstruction quality for different wave numbers $\gamma^{\star}$, we focus on the z-axis separability and detectability. The z-axis separability is the ability to separate two tumours along the z-axis. It has been calculated from the reconstructed concentrations of tumour \# 2 and \#3. The z-axis separability is defined as the difference between the peak and valley reconstructed concentrations as specified on figure 3. The z-axis detectability is defined as the reconstructed concentration of the tumour \#1. On figure 4, the z-axis separability and detectability are plotted with respect to $\gamma^{\star}$ for three maximum numbers of detected photons.

It can be seen that the parameter $\gamma^{\star}$ impacts the reconstruction quality as 

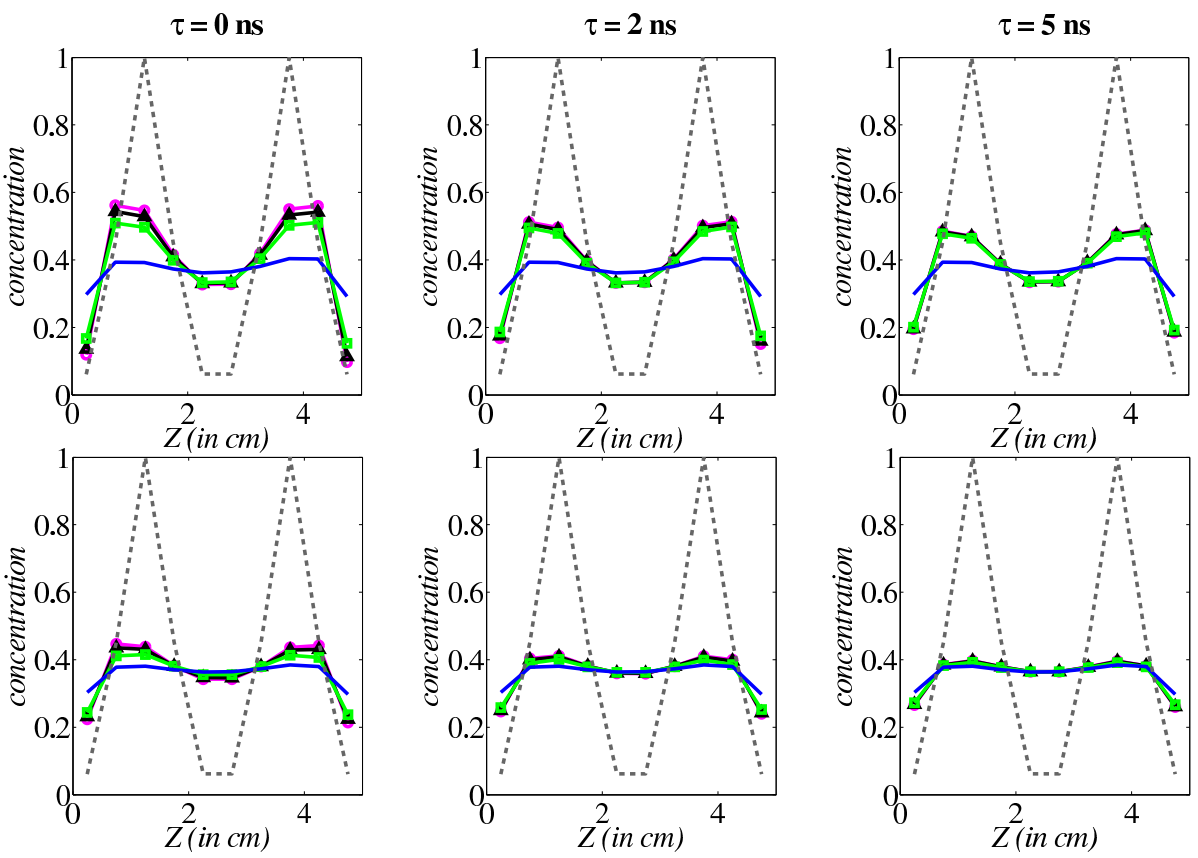

Figure 5. Reconstruction profiles along the z-axis, through tumour \#2 and \#3, for three fluorescence lifetimes. The dashed line indicate the ground truth concentration profile. In the first column $\tau=0 \mathrm{~ns}$, in the second $\tau=2 \mathrm{~ns}$, and in the $\tau=5$ ns. $C_{\max }$ is set to $10^{10}$ (top row) and to $10^{8}$ (bottom row). Here again, $\mu_{a}=0.1 \mathrm{~cm}^{-1}, \mu_{s}^{\prime}=10 \mathrm{~cm}^{-1}$. Moments are used up to order 0 (blue solid line), 1 (green marked with $\square$ ), 2 (black marked with $\triangle$ ) and 3 (magenta marked with $\bigcirc)$.

predicted. For a given SNR, the z-axis separation and detection are better within a medium of large $\gamma^{\star}$ than within a medium of small $\gamma^{\star}$.

\subsection{Influence of the fluorescence lifetime of the markers}

In this last part, the influence of the fluorescence lifetime with respect to the reconstruction quality is evaluated. We have predicted the benefit of using higher order moments to be decreasing when increasing fluorescence lifetimes are considered. To verify this prediction, reconstructions profiles along tumour \#2 and \#3 are plotted on figure 5 for the three cases $\tau=0$ ns, $\tau=2 \mathrm{~ns}$, and $\tau=5$ ns (left, middle, and right columns). The maximum number of detected photons has been set to $C_{\max }=10^{10}$ and $C_{\max }=10^{8}$ (top and bottom rows).

The degrading effect of the lifetime is observed on reconstructions for which $C_{\max }$ is set to $10^{8}$ photons. Indeed, it can be observed on the bottom row of figure 5 that the reconstruction can be improved by addition of the $m_{1}$ 's when $\tau=0$ ns while the benefit provided by the $m_{1}$ 's is severely limited when $\tau$ is set to 5 ns. Moreover, we have verified (results not shown) that the degradation due to the lifetime is amplified for media with large mean speed of detected photons $v^{\star}=2 c\left(\mu_{a}\right)^{1 / 2} /\left(3 n \mu_{s}^{\prime}\right)^{1 / 2}, c$ being the speed of light in vacuum. These two points are in agreement with the predictions of the first paper of this series. However, when $C_{\max }=10^{10}$ photons, the quality of the reconstructions remains about the same, even for a lifetime as long as 
5 ns photons.

To understand these observations, let us get back to the first part of this work in which it was observed that the fluorescence first order moment consists of a desirable marker dependant component and an undesirable marker free component. The marker free component was shown to get larger and larger for increasing fluorescence lifetime. Therefore, when the fluorescence lifetime is too large, the marker free component overwhelms the marker dependent component. This explains why the reconstruction quality decreases with increasing fluorescence lifetime. Nevertheless, the marker dependent component may remain significant with respect to the measurement signal even if it is much lower than the marker free one - provided that the measurement can be performed accurately enough. It can be thought the accuracy of the measurement is large enough for $C_{\text {max }}=10^{10}$ photons to avoid the degrading effects of the marker lifetime.

\section{Conclusion}

In this paper, 3-D reconstructions of the markers concentration are performed from the moments of synthetic measurements up to different orders. The reconstruction methodology we used is based on the classical Tikhonov regularization implemented via SVD, together with an original procedure for choosing the regularization parameter. This methodology guarantees the reconstructions to be rigorously comparable and may be profitable in other contexts - for instance the optimization of the optode arrangement or the evaluation of the influence of the the forward model.

The main result of the present study is to show that higher order moments are only beneficial when the SNR of the measurements is large enough. Specifically, we observe that higher order moments start being profitable when more than $10^{8}$ photons can be detected. Bellow this limit, the higher order moments do not improve the reconstruction quality. Therefore, using only the $0^{\text {th }}$ order moments is sufficient. This result is not surprising in the sense that the higher order moments were previously identified to be strongly correlated and corrupted by noise in the first part of this work. However, the value of the limit is surprisingly high and can be hardly reached in some experimental situations. As far as the absorption reconstruction in DOT is concerned, the $10^{8}$ photons limit can be reached within tractable integration times provided that the medium is not too thick and/or absorbing. However, as far as FDOT is concerned, the number of detected photons is much lower due to losses mainly related with the small absorption cross section of the fluorescence markers but also with the photons conversion (quantum yield). Typically, the amplitude of fluorescence signals are about $10^{4}$ times smaller than their diffuse counterparts. Moreover, if the rate of detected photons would reach a too low level - typically some tens of photon counts per second for a cooled photomultiplier tube, then the electronic noise could no longer be neglected and the limit of $10^{8}$ photons should be raised.

The optical properties of the medium and the fluorescence lifetime of the marker are found to have a much lower impact on the reconstruction quality. However, this confirms the conclusions of the first part concerning the influence of the parameter $\gamma^{\star}$ when the lifetime is zero and of the parameters $v^{\star}$ when the lifetimes is non-zero.

More conclusively, the moment method turns out to be dramatically limited by the presence of intrinsic photon noise on the time-resolved measurements. This drawback could prevent its application to FDOT for which the number of detected photons - and thus the SNR - is quite low. Alternative features such as Laplace 
transform (Gao et al., 2006), full width at half maximum, peak value, time at peak value (Riley et al., 2007) or number of photons within time windows (Grosenick et al., 2005) have been proposed. Further comparative studies still have to be engaged to determine if such features could overcome the limitations identified in this study.

\section{Acknowledgments}

This work has been supported by the Région Rhône-Alpes in the context of project I3M 'Multiscale Medical Imaging and modelling: from the small animal to the human being' of cluster 'Informatique, Signal, Logiciel Embarqué'.

\section{References}

\section{References}

Bertero, M., Mol, C. D., and Pike, E. R. (1988). Linear inverse problems with discrete data: II. stability and regularisation. Inverse Probl., 4(3):573-594.

Cheong, W. F., Prahl, S. A., and Welch, A. J. (1990). A review of the optical properties of biological tissues. IEEE J. Quantum Electron., 26(12):2166-2185.

Culver, J. P., Choe, R., Holboke, M. J., Zubkov, L., Durduran, T., Slemp, A., Ntziachristos, V., Chance, B., and Yodh, A. G. (2003). Three-dimensional diffuse optical tomography in the parallel plane transmission geometry: Evaluation of a hybrid frequency domain/continuous wave clinical system for breast imaging. Med. Phys., 30(2):235-247.

Ducros, N., Silva, A. D., Dinten, J.-M., and Peyrin, F. (2009). A comprehensive study of the use of temporal moments in time-resolved diffuse optical tomography: Part i. theoretical material. Phys. Med. Biol. .

Galatsanos, N. and Katsaggelos, A. (1992). Methods for choosing the regularization parameter and estimating the noise variance in image restoration and their relation. IEEE Trans. Image Process., 1(3):322-336.

Gao, F., Tanikawa, Y., Zhao, H., and Yamada, Y. (2002). Semi-three-dimensional algorithm for time-resolved diffuse optical tomography by use of the generalized pulse spectrum technique. Appl. Opt., 41(34):7346-7358.

Gao, F., Zhao, H. J., Tanikawa, Y., and Yamada, Y. (2006). A linear, featured-data scheme for image reconstruction in time-domain fluorescence molecular tomography. Opt. Express, 14(16):7109-7124.

Gaudette, R. J., Brooks, D. H., Di Marzio, C. A., Kilmer, M. E., Miller, E. L., Gaudette, T., and Boas, D. A. (2000). A comparison study of linear reconstruction techniques for diffuse optical tomographic imaging of absorption coefficient. Phys. Med. Biol. , 45(4):1051-1070.

Golub, G., Heath, M., and Wahba, G. (1979). Generalized cross-validation as a method for choosing a good ridge parameter. Technometrics, 21(2):215-223.

Graves, E. E., Culver, J. P., Ripoll, J., Weissleder, R., and Ntziachristos, V. (2004). Singular-value analysis and optimization of experimental parameters in fluorescence molecular tomography. J. Opt. Soc. Am. A, 21(2):231-241. 
Grosenick, D., Moesta, K. T., Moller, M., Mucke, J., Wabnitz, H., Gebauer, B., Stroszczynski, C., Wassermann, B., Schlag, P. M., and Rinneberg, H. (2005). Time-domain scanning optical mammography: I. recording and assessment of mammograms of 154 patients. Phys. Med. Biol. , 50(11):2429-2449.

Hansen, C. (1987). The truncated svd as a method for regularization. BIT Num. Math., 27(4):534-553.

Hansen, C. (1992). Analysis of discrete ill-posed problems by means of the l-curve. SIAM Rev., 34(4):561-580.

Hyde, D., Miller, E., Brooks, D., and Ntziachristos, V. (2007). A statistical approach to inverting the born ratio. IEEE Trans. Med. Imaging, 26(7):893-905.

Kepshire, D. S., Davis, S. C., Dehghani, H., Paulsen, K. D., and Pogue, B. W. (2007). Subsurface diffuse optical tomography can localize absorber and fluorescent objects but recovered image sensitivity is nonlinear with depth. Appl. Opt., 46(10):16691678.

Kilmer, M. E. and O'Leary., D. P. (2001). Choosing regularization parameters in iterative methods for ill-posed problems. SIAM J. Matrix Anal. Appl., 22(4):12041221.

Kumar, A. T. N., Raymond, S. B., Dunn, A. K., Bacskai, B. J., and Boas, D. A. (2008). A time domain fluorescence tomography system for small animal imaging. IEEE Trans. Med. Imaging, 27(8):1152-1163.

Lam, S., Lesage, F., and Intes, X. (2005). Time domain fluorescent diffuse optical tomography: analytical expressions. Opt. Express, 13(7):2263-2275.

Medintz, I. L., Uyeda, H. T., Goldman, E. R., and Mattoussi, H. (2005). Quantum dot bioconjugates for imaging, labelling and sensing. Nat. Mater., 4(6):435-446.

Miller, K. (1970). Least squares methods for ill-posed problems with a prescribed bound. SIAM J. Math. Anal., 1(1):52-74.

Nielsen, T., Brendel, B., Ziegler, R., van Beek, M., Uhlemann, F., Bontus, C., and Koehler, T. (2009). Linear image reconstruction for a diffuse optical mammography system in a noncompressed geometry using scattering fluid. Appl. Opt., 48(10):D1D13.

Regińska, T. (2004). Regularization of discrete ill-posed problems. BIT Num. Math., 44(1):119-133.

Riley, J., Hassan, M., Chernomordik, V., and Gandjbakhche, A. (2007). Choice of data types in time resolved fluorescence enhanced diffuse optical tomography. Med. Phys., 34:4890.

Selb, J., Dale, A., and Boas, D. (2007). Linear 3d reconstruction of time-domain diffuse optical imaging differential data: improved depth localization and lateral resolution. Opt. Express, 15(25):16400-16412.

Tikhonov, A. N. and Arsenin, V. A. (1977). Solution of Ill-posed Problems. WinstonWiley, New York.

Urano, Y., Asanuma, D., Hama, Y., Koyama, Y., Barrett, T., Kamiya, M., Nagano, T., Watanabe, T., Hasegawa, A., Choyke, P. L., and Kobayashi, H. (2009). Selective molecular imaging of viable cancer cells with ph-activatable fluorescence probes. Nat Med, 15(1):104-109. 